
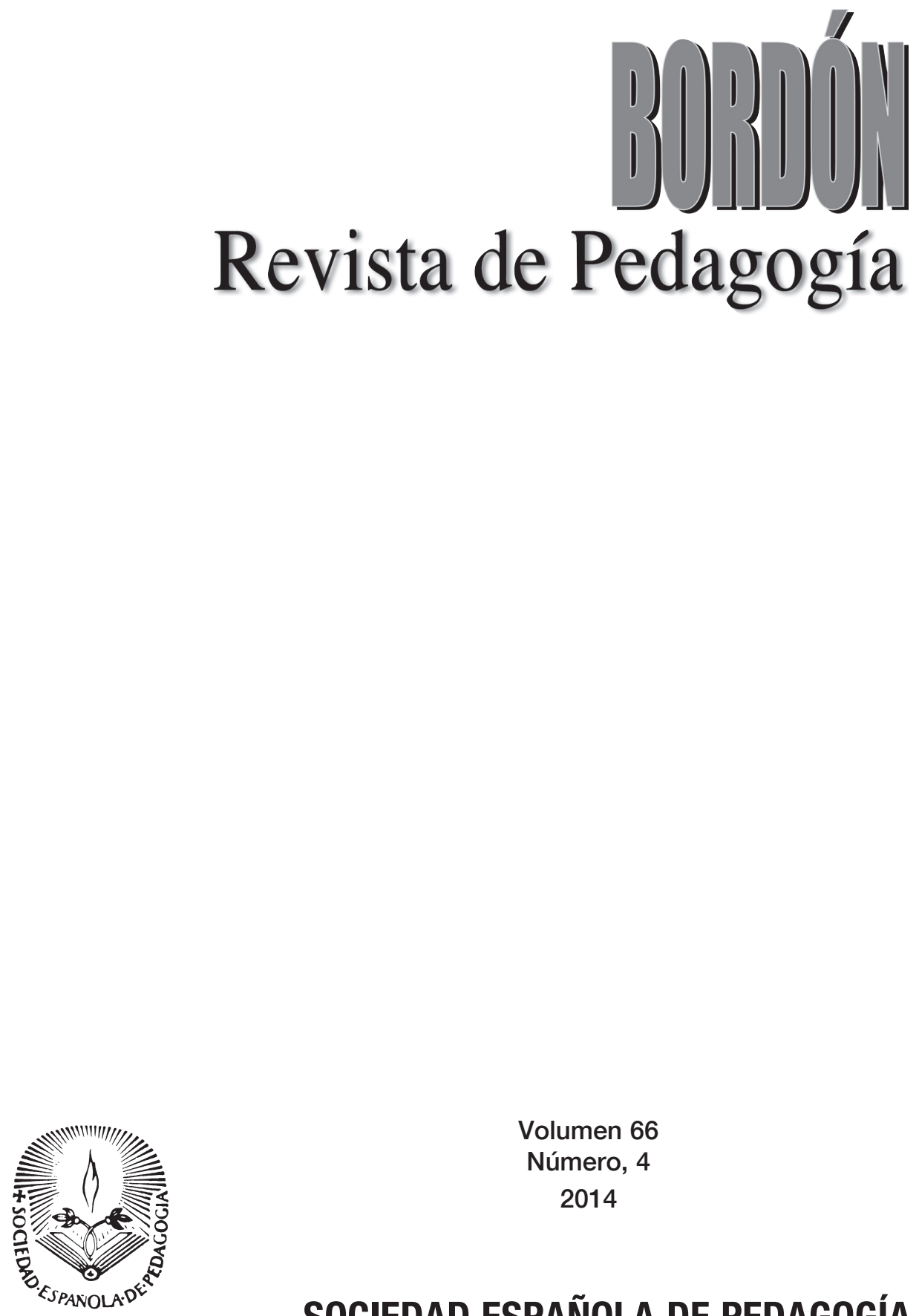

Volumen 66

Número, 4

2014

SOCIEDAD ESPAÑOLA DE PEDAGOGÍA 


\title{
ESCRIBO COMO HABLO. LAS IDEAS PEDAGÓGICAS DE GONZALO CORREAS
}

\section{I write as I talk. Pedagogical ideas of Gonzalo Correas}

\author{
ALEJANDRO GÓMEZ CAMACHO
}

Universidad de Sevilla

D01: 10.13042/Bordon.2014.66405

Fecha de recepción: 26/09/2013 - Fecha de aceptación: 27/02/2014

Autor de contacto / Corresponding Author: Alejandro Gómez Camacho. Email: agomez2I @ us.es

INTRODUCCIÓN. Gonzalo Correas es considerado como uno de los más importantes humanistas del Siglo de Oro español; la Ortografia kastellana nueva i perfeta es su obra más afamada, e incluye una gran cantidad de referencias educativas. Este trabajo analiza las ideas pedagógicas en la obra de Correas, así como las relaciones que establece entre la ortografía fonética y la enseñanza de la lectura. MÉTODO. Se recurre para ello al análisis comparado de las obras de Correas y de las referencias a la educación en otras ortografías españolas del siglo XVII que utilizaron argumentos pedagógicos, interesadas todas ellas en una norma ortográfica que facilitase el aprendizaje infantil a pesar de que mantenían propuestas irreconciliables en la escritura. RESULTADOS. Al estudiar las referencias a la educación, nos encontramos con una idea recurrente: el español necesita un nuevo alfabeto que refleje la realidad de la pronunciación en el siglo XVII. Las nuevas letras ayudarían a los niños a aprender la lectura de una forma más adecuada y con mayor celeridad; Correas cambia incluso los nombres de las letras y el orden del alfabeto para facilitar el aprendizaje. Consecuentemente, se propone también un nuevo silabario. DISCUSIÓN. Los resultados de este trabajo muestran que en el primer tercio del Seiscientos, el argumento pedagógico se ha incorporado definitivamente a la controversia sobre la reforma de la ortografía española. Estas obras, además de su valor lingüístico y filológico, ofrecen un valiosísimo testimonio sobre cómo se concebía la alfabetización en nuestro Siglo de Oro que hasta hoy no ha sido estudiado.

Palabras clave: Gonzalo Correas, Ortografía española, Historia de la educación, Enseñanza de la lectura, Siglo XVII. 


\section{Introducción}

El maestro Gonzalo Correas es uno de los humanistas más relevantes del siglo XVII, tanto por sus estudios sobre gramática y ortografía como por su labor de paremiólogo que le llevó a coleccionar refranes, proverbios y canciones. Sin embargo, en la prolija bibliografía sobre el catedrático de Salamanca apenas presta atención a las abundantes referencias al aprendizaje y a la enseñanza de la lengua española, sobre todo en el contexto de la radical apuesta por una ortografía fonética en la que se utiliza una única letra para cada sonido del español y en la que cada letra solo representa un sonido en la escritura; rompía así definitivamente con la tradición de impresores, escribanos y maestros que utilizaban el alfabeto de origen latino por motivos etimológicos y consagraban dificultades ortográficas arbitrarias, muchas de las cuales aún hoy perviven en nuestra lengua.

Gonzalo Correas tuvo un inmenso prestigio como filólogo en su siglo, tanto por sus textos sobre las lenguas clásicas como por la defensa apasionada de la lengua española en sus obras mayores; en este estudio nos ocuparemos de las referencias al aprendizaje de la lectura y de la escritura que aparecen en sus propuestas ortográficas formuladas durante los últimos años de su vida. En la introducción de Manuel Taboada al Arte kastellana (1984) se incluye un catálogo exhaustivo de su obra que muestra el ingente trabajo de nuestro autor sobre las lenguas y su enseñanza; no nos interesan en este estudio los aspectos lingüísticos y filológicos de la misma, sino la mirada atenta de un profesor al modo como los niños se acercaban al aprendizaje de la lectura y la escritura en su lengua materna.

Correas crea un nuevo alfabeto basándose en la autoridad de los dos autores más relevantes que propusieron la reforma fonética de nuestra escritura "al mui doto maestro de España el Antonio de Nebrixa, ó Lebrixa, i al grande inxenio tanbien Andaluz Mateo Aleman“ (1630: 4), cuyas reformas considera imperfectas e incompletas. En consecuencia, reduce a veinticinco las letras españolas suprimiendo tildes, dígrafos y todas las letras en su opinión innecesarias; por ejemplo, $c, c, j$, qu o $y$, pero mantiene paradójicamente $b$ y $v$ que carecían de distinción fonológica. Utiliza nuevas tipografías para las letras $a, d, v$ y $k$, que recuerdan al alfabeto griego, y crea nuevas letras ligadas para los dígrafos $r r$, ch y $l l$ en mayúscula y en minúscula; también unifica la $\tilde{n}$ en un único signo sin tilde.

Para facilitar la memorización de los niños, reordena el alfabeto y agrupa las letras en vocales, finales de sílaba, liquidantes y antevocales; no se basa en los rasgos articulatorios sino en la combinatoria silábica. Lo que tradicionalmente se consideraba como un inconveniente se convierte así en su principal virtud; Correas no pretende ser un fonetista sino un pedagogo (salvando el anacronismo de los términos), no pretende describir el sistema de la lengua sino facilitar el aprendizaje de la lectura. En el mismo sentido, los nombres de las letras se reducen a monosílabos: $a, e, i, o, u, r e, l e, n e, s e, z e$, $x e$, de, fe, ga, be, ka, pe, te, va, me, rra, che, lla, $\tilde{n} e, h a$.

"De la orden ke deven tener las letras en el Abeze ninguno á tratado ni se akordó, siendo lo ke mas inporta para la fazilidad de aprender, i enseñar á leer. Aki las pondremos por orden por sus klases i dinidad: i las kolokaremos kada una en el lugar ke la konpete, no konfusas i arroxadas akaso, komo estavan antes: Primero las zinko vokales, luego siete ke son finales, despues otras siete ke son likidantes, al fin seis ke son antevokales. Llegemos á eskoxerlas. Las demas letras desta klase, i de las otras, no inporta estar antes ú despues unas de otras, en prinzipio, medio, ó fin: fuera de las ke noto por partikular rrazon, i konvenienzia, atendiendo á la maior fazilidad, i komodidad de enseñar á leer, i deprender"(1630: 68). 
En el Arte de la lengua española castellana identifica expresamente las sílabas con la lectura "en Rromanze se llaman partes en el deletrear, porque dezimos del niño que comienza á saber leer, que ia sabe xuntar las partes, que es las silabas" (1954: 117), y se ofrece una propuesta pedagógica concreta para aprender a leer con un método silábico renovado.

"Ase de enseñar de zinko en zinko silabas por el numero de las vokales, i hazerlas rrepetir de memoria, i de memoria se an de enseñar: i luego mostrar las letras komo lo dizen. I el niño kaerá presto en la kuenta, i diskurrirá por todas las klases dichas. I en estando fazil en ellas, á de leer de leido. I kuando dudare en alguna parte, ke la buelba á buscar á estos Beabaes, i enseñarle komo lo á de hazer" (1630: 86-87).

También se propone un nuevo silabario sujeto al mismo orden y a los mismos principios; así afirma que "pudieranse poner mas silabas posibles [...] mas para enseñar estas bastan" (1630: 86); de nuevo se estudia cuidadosamente el orden para facilitar el aprendizaje "es bien seguir la orden por las ke son mas usadas" (1630: 86) de tal forma que las sílabas aprendidas en primer lugar permitan inferir con facilidad las posteriores y la formación de palabras.

Por motivos tipográficos no reproducimos el "Abeze" castellano que propone Correas en su ortografía (1630: 65): ocho de las veinticinco letras necesitarían caracteres especiales en minúscula y cuatro más en mayúscula; pero no podemos olvidar que el catedrático salmantino utiliza en la práctica la ortografía que propone (el impresor Tabernier funde nuevos tipos de imprenta para dar a las prensas la Ortografia kastellana nueva i perfeta) por lo que sus textos ofrecen una apariencia muy singular y son de difícil lectura, como se puede comprobar fácilmente en la versión digitalizada de acceso libre de la Biblioteca Nacional de España. Por este motivo, el alfabeto de Correas no vuelve a utilizarse en la práctica en ningún texto más allá de esta obra. De origen humilde, Gonzalo Correas se dedicó toda su vida a la enseñanza en la Universidad de Salamanca (Alarcos, 1919) y, según señala Taboada en su introducción al Arte kastellana, "su metodología docente refleja una coherente pedagogía que era alabada por sus alumnos" (1984: 27). Para la enseñanza de las lenguas clásicas, propuso un novedoso método en el que se partía del conocimiento profundo del español y de lo que las lenguas tienen en común, antes de abordar el estudio del latín y del griego (Bustos Tovar, 1998); pero la preocupación por la enseñanza de las lenguas tiene en Correas una perspectiva muy amplia y con frecuencia se inicia en los niños que aprenden a leer y a escribir.

"porque sienpre me parezió, desde que tuve esperienzia de enseñar estas lenguas, que se á de comenzar por la gramatica vulgar, que á de andar al prinzipio de la Latina, i que los niños comienzen á leer i escrivir por ella en las escuelas, i que esta comienze por el Cristos, i letras, i beabaes que podemos llamar silabas, como lo son, i en sabiendo aquel prinzipio de letras, ó cartilla, que prosigan leiendo de leido por las rreglas, i partes de la gramatica de su lengua materna, i luego pasen á la arte de Latin, de la manera que io lo dispongo en esta" (1954: 9).

En otras palabras, la preocupación por la infancia y por su forma de aprender no está presente solo en las ortografías, sino que son el punto de partida de la monumental obra filológica del catedrático de Salamanca; en un sentido amplio, podríamos afirmar que también el criterio pedagógico fundamenta la principal seña de identidad de su obra: la prioridad del español sobre el latín; en la línea que se observaba en las gramáticas de español para otros reinos (Esteba, 2005) y en las gramáticas escritas para los nativos del nuevo mundo (Lope, 1997). Cierto es que en los manuales de educación del Siglo de Oro hay una corriente que prescinde de la gramática latina como punto de partida (Laspéras, 1995); pero es en la obra de Gonzalo 
Correas donde esta tendencia se manifiesta con más claridad, a pesar de que era un profesor de lenguas clásicas y de que su ingente biblioteca se ocupa sobre todo de la enseñanza de las mismas (Rodríguez San Pedro, 1986).

Gonzalo Correas se ocupa de la ortografía fonética y por extensión de las ideas sobre el aprendizaje que se asocian a la misma en tres de sus obras: el Arte de la Lengua Española Castellana (1625), Trilingue de tres artes de las tres lenguas Castellana, Latina i Griega, de la que el Arte kastellana es parte (1627), y sobre todo en la Ortografia kastellana nueva i perfeta (1630).

Las propuestas del maestro Correas culminan los intentos de establecer una ortografía fonética en nuestro Siglo de Oro (Galán Melo, 1992) y provocan una fortísima reacción etimológica en defensa de la tradición escrita de impresores, secretarios y maestros, descrita por Gómez Camacho (1992) en su edición de la ortografía de Juan de Robles. Los continuos cambios en la pronunciación de nuestra lengua que se originan por el reajuste consonántico del español durante los siglos XVI y XVII propiciaron la publicación de decenas de ortografías en defensa de un nuevo sistema de escritura que se adecuara al viejo principio del escribo como hablo; se trataba de suprimir las letras innecesarias heredadas del latín para reproducir cada fonema con un único grafema (cada sonido con una letra), una ortografía pretendidamente fonética que aún hoy cuenta con defensores en el ámbito hispánico. Estas propuestas innovadoras nunca llegaron a aplicarse en su totalidad; pero influyeron decisivamente en la ortografía académica del siglo XVIII que aquilata lo que hoy reconocemos como norma culta del español escrito (Bustos Tovar, 62). El origen de las propuestas de una reforma fonética de la ortografía se remontan a la primera gramática española del sevillano Antonio de Nebrija en su Gramatica castellana (1492) y sobre todo en las Reglas de ortographia (1517); esta tendencia se radicalizó en Fernando de Herrera y en Mateo Alemán, y llega a su extremo en la obra de Correas (Cavillac, 1980; Paz, 2002).

Nuestro autor se siente efectivamente la culminación de una corriente imparable en español. En el último capítulo de la ortografía, la euforia Correas se desborda rozando el delirio, así sueña (como ya apuntaba en la dedicatoria) que apenas se publique su obra todos abrazarán entusiasmados su ortografía y que el rey Felipe IV será un nuevo Alfonso X por imponer en escuelas e imprentas la escritura que propone.

"Mas si otros kuriosos me aiudan, komo espero, ke los ái kuerdos, i deseosos, ke no esperan á mas de ke io komienze a inprimir, mui presto se introduzirá. I mas si el Rrei nuestro Señor, enterado dello lo mandase exekutar en las enprentas, i poner en la kartilla el Abeze rrenovado, daria por akabada esta enpresa. I no me kedará por intentar este medio: ke es bastante á hazer inmortal á un Rrei en la fama" (1630: 46).

Ni que decir tiene que la peculiar ortografía de Correas originó una reacción en defensa de la tradición que se inicia inmediatamente en $\mathrm{El}$ culto sevillano de Juan de Robles y culmina con las ortografías académicas del siglo XVIII. Aunque las propuestas de Correas no tuvieron repercusión práctica alguna, Esteve Serrano (2007) y Martínez Marín (1992) coinciden en que sus esfuerzos contribuyeron decisivamente a la modernización de la ortografía española tras el reajuste consonántico de nuestro idioma.

La ortografía de Correas es un ejemplo extremo de la paradoja que con tanto acierto señalaba Johnston (1988) sobre la ortografía de Mateo Alemán: la búsqueda de la completa legibilidad produce un texto ilegible. A pesar de la apariencia tan singular que ofrecen al lector las citas de las obras de Gonzalo Correas que se insertan en este trabajo, la ortografía se ha modernizado en todos aquellos casos en que es imposible reproducir el original; por ejemplo, en los dígrafos y en las letras que Correas 
inventa. También se han regularizado las mayúsculas, las eses altas o las abreviaturas; pero el resultado dista mucho de la extrema facilidad de lectura que el humanista extremeño supone a la nueva forma de escritura.

Desde un punto de vista filológico, las ortografías fonéticas del siglo XVII fracasaron en sus propuestas porque rompían la unidad de la escritura con el latín y con otras lenguas europeas, y porque en la práctica la utilización de nuevas letras ajenas al español dificultaban en extremo la lectura y la escritura en nuestra lengua. Sin embargo, desde el punto de vista de la enseñanza de la lectura y de la escritura, el principio del "tenemos de eskrivir, komo pronunziamos: é pronunziar, komo eskrivimos" (Correas, 1630: 14) buscaba facilitar el acceso a la comunicación escrita, por lo que de manera natural llevó a una reflexión sobre su aprendizaje "den lugar á los niños para komenzar i aprender por lo mexor, i del todo perfeto" (Correas, 1630, 3v).

En las ortografías de Mateo Alemán y de Gonzalo Correas se reflexiona sobre el aprendizaje conjunto de la lectura y la escritura, la simplificación del alfabeto o el uso de silabarios como recurso didáctico para la alfabetización (Gómez Camacho, 2014); se favorece así la renovación del método de enseñanza que partía de la memorización de los nombres de las letras del alfabeto latino, lo que culminará a finales del siglo siguiente con la adopción del método silábico y el aprendizaje simultáneo de la lectoescritura (Viñao, 1992; Ruiz Berrio, 2004; Ortega, 2011).

Si en la historia de la ortografía española estas obras constituyeron una propuesta fallida, merecen, sin embargo, un lugar relevante entre las obras que se ocuparon de la educación en el siglo XVII. La vinculación de la ortografía fonética con la renovación de las prácticas pedagógicas referidas a la escritura perdurará en el tiempo, y será uno de los factores que determinan el fracaso de nuevos intentos de reforma de la ortografía española en los países americanos durante los siglos XVIII y XIX (Frago, 2012).

\section{La influencia de las ideas pedagógicas de Mateo Alemán}

Mateo Alemán es el autor español que se cita con más frecuencia en la Ortografia castellana nueva i perfeta de Correas; es el modelo que se sigue y al que se recurre como fuente de autoridad. De hecho el catedrático de Salamanca insiste en que las abundantísimas coincidencias entre ambas obras no son fruto de la imitación, puesto que ya había elaborado sus teorías antes de leer las de Alemán, y no desaprovecha la ocasión de señalar algún defecto en la obra del sevillano.

"Aleman anduvo en esto azertadisimo, i konvinieramos kasi en todo, sin saber uno de otro, si el no eszediera en añedir zinko letras de mas de la mesma boz i sonido de otras, ke fue superfluo. Kuando el inprimio en Mexiko, eskrivia ió aká en linpio lo ke antes tenia forxado, ke á veinte años, i avrá tres ke llego á mi notizia, i mano su ortografia“ (1630: 63).

Sin embargo, se lamenta sinceramente de no haber conocido al autor del Guzmán de Alfarache cuando trata de las letras "En esto advirtio mui bien Mateo Aleman, ansia tengo de no averle podido komunikar: ke konvenimos en mucho, i en lo sustanzial de los nombres" (1630: 53). Desde el prólogo se le cita como autoridad junto a Antonio de Nebrija "al grande inxenio tanbien Andaluz Mateo Aleman, ke eskrivió ortografia el ultimo, i la inprimió en méxico" (1630: 4).

También recuerda a Mateo Alemán en su Arte de la lengua española castellana "i despues Mateo Aleman, con muchas advertencias nuevas i mui acertadas" (1954: 8) e incluso llega a considerar al Guzmán de Alfarache como un modelo literario del español de su época en el uso de refranes 
y frases hechas a las que Correas era tan aficionado "I provaran tanbien la dificultad, si traduxeran el libro del Picaro Guzman de Alfarache de Mateo Aleman, que usa mucho dellas, i es inposible traduzirle bien en otra lengua" (1954: 489). Esta referencia cobra especial relevancia si consideramos que Parrack propone un principio pedagógico en la "escuela picaresca de aprendizaje" (2005: 300) que Gómez Canseco (2012) desarrolla en detalle en la introducción a su edición académica de la obra.

En la Ortografia kastellana nueva i perfeta se recurre con mucha frecuencia al sevillano; basta la invocación a su autoridad para que Correas dé sus propuestas por válidas, por muy descabelladas que sean. "M. Aleman advirtió bien, i kon buenas razones" (1630: 15), "advierte bien Alemán" (1630: 9), "konociendo kon su grande inxenio ke inportava" (1630: 35), etc.

La Ortografía castellana (1609) de Mateo Alemán cuenta entre sus méritos la formulación de una propuesta pedagógica muy original que se anticipa en dos siglos a la que triunfaría en España (Chartier, 2001). En esta obra se dedica expresamente el capítulo segundo a la reflexión sobre cómo se enseñaba y se aprendía la lengua española y su ortografía; su título resume a la perfección la intención de la nueva ortografía: "De la inorancia de los maestros pasados, i cuánto importa la enmienda en los presentes, facilitando el escrevir ortógrafamente" (1950: 21).

La originalidad de la ortografía de Mateo Alemán no reside solo en la atrevida propuesta de reforma fonética y en la invención de nuevas letras, sino en que es el primer autor que concede una importancia capital a la enseñanza como un elemento imprescindible en la difusión de la nueva forma de escribir (Gómez Camacho, 2014). En la obra de Alemán, además de la preocupación constante por la infancia, y de los consejos a los buenos maestros y la censura de los maestros incompetentes, encontramos propuestas pedagógicas muy innovadoras. De hecho es la primera obra que propone la enseñanza y el aprendizaje de la lectura simultáneamente con la escritura, una práctica que no se generalizaría en nuestras escuelas hasta finales del siglo XVII por intereses gremiales y por el elevado coste de los materiales necesarios para la escritura, en opinión de Viñao (1999) y Quetgles Roca (2004).

"Bolviendo a propósito del buen método para escribir, pregunto [...] si el escrevir es un modo de dibujar, diferentísimo de leer, qué inconveniente se sigue que los niños aprendan uno i otro juntamente, como se les pudiera enseñar, leer i tañer, escrevir i dançar, o cualesquiera otras dos artes distintas? Mi parecer es, aunque se les haga duro á otros, i más á los maestros, que pues no son cosas que impide ó haze contradición saberse juntas" (1950: 25).

Además de la propuesta de la lectoescritura frente a la tradición de los maestros calígrafos del siglo XVI, Alemán postula planteamientos tan novedosos para su época como la adecuación de la materia a la edad y las capacidades de los niños.

"I así, cuando se trata dellos, deven los buenos maestros considerar dos cosas, de qué calidad sea lo que nos enseñan, y qué capacidad la del sujeto á quien lo enseñan. Diferentemente recibe la doctrina un muchacho de diez años, que otro de cuatro; i con mayor facilidad, cualquiera dellos vendrá en saber tejer, que á fabricar un relox; coser un çapato, que misturar un órgano: i nada se iguala, con lo dificultoso de las letras" (1950: 21).

También censura la enseñanza de la escritura a los niños con caligrafías elaboradas y adornos innecesarios, y propone un único tipo de letra manuscrita sin "potajitos de formas" (1950: 24) que dificulten el aprendizaje.

En nuestra opinión, la influencia de Mateo Alemán en Gonzalo Correas no se limita a la ortografía; también se extiende a lo que podríamos 
denominar sus ideas pedagógicas, sobre todo en la importancia que se concede a la educación como un elemento imprescindible en los intentos de reforma ortográfica. En ambos autores, la propuesta ortográfica y pedagógica comparten el mismo principio: la superación de la tradición y el establecimiento de un nuevo sistema que se adecue a los cambios que está sufriendo la lengua en el Seiscientos.

\section{El criterio pedagógico en la ortografía fonética}

Como se ha apuntado anteriormente, el deseo de una ortografía que reproduzca cada sonido con una única letra se fundamenta, entre otros motivos, por la facilidad del aprendizaje de la lectura y de la escritura en los niños que acceden al lenguaje escrito. Toda la ortografía se volvería así supuestamente natural y se oiría en la lengua oral; la lectura y la escritura en español se limitarían en la hipótesis de estos ortógrafos a la transposición mecánica y sin arbitrariedades de la pronunciación. El primer autor que incorpora el argumento pedagógico desarrollado y recurrente en su ortografía es, ya lo hemos visto, Mateo Alemán, y Gonzalo Correas sigue el modelo del sevillano desde su peculiar visión del problema. Sin duda, el principal mérito de Correas es su empeño por observar la realidad y adecuarse a ella, ya sea en las partes de la oración (Hernando, 2010), en la ortografía o en la enseñanza de la lectura.

En la dedicatoria de la Ortografía kastellana nueva i perfeta al rey Felipe IV se enuncia expresamente el principio pedagógico que anima a la obra y se avala con la experiencia docente de Correas. La obra se dedica también a un niño, el príncipe Baltasar Carlos, a quien se ofrece un camino fácil para aprender a leer; Correas imagina que la autoridad real "mandará ke estas letras escoxidas se pongan en la kartilla komun".

"Desta menor parte de letras primeras, aunke grande, por ser fundamento de las maiores, hago presente á V. A. para ke le sea fazil en los tiernos años la letura, sin la molestia vulgar. Komo esperimentado en esta materia por otras lenguas, é notado i eskoxido las letras, ke avemos menester para eskrivir perfetamente en nuestra lengua Kastellana, i dispuestolas en orden i fazilidad kon su deletreazion para deprender i enseñar" (1630: V).

El catedrático de Salamanca, ya a las puertas de la muerte, no es consciente del disparate ortográfico que propone en su afán por desligar la escritura del español de la influencia del latín; pero no cabe duda de que el argumento que destaca ante el rey y el príncipe heredero de España es claramente pedagógico: el beneficio que comporta para aprender y enseñar la lectura y la escritura del español. La preocupación por la infancia es constante, dado que su obra se escribe "atendiendo á la maior fazilidad, i komodidad de enseñar á leer, i deprender" (1630: 68), y contrasta con otras preceptivas que se dirigen expresamente a los nobles (Baranda, 1955). Así exclama en defensa de la ortografía fonética que propone: "Kien puede aver tan inkonsiderado por lo ke á sí toka, pues á el se le dexa libertad de estarse en su uso, ke no kiera, ke los niños le deprendan mas fazil i mexor?" (1630: 46).

La gran aportación de la ortografía de Correas es, sin duda, un nuevo alfabeto para el español que se ajusta de forma perfecta, en opinión de su autor, al principio fonético de representación de cada fonema con un único grafema. También en este caso se invoca el principio pedagógico para justificar una propuesta tan radical y tan llamativa para cualquier lector.

"El rremedio uniko es rrenovar lo viexo, i no ái otro, dexar el abuso, i tomar uso nuevo. Kuanto mas fazil será al niño, i al maior, saber solas veinte i zinko letras, ke le daremos, para leer i eskrivir en Kastellano perfetamente, ke tanto enbarazo?" (1630: 42). 
Este argumento se convierte incluso en una obligación patriótica para Correas, que en su Arte de la lengua española castellana considera que facilitar la lectura y la escritura a niños y jóvenes no es sino una muestra de amor a su patria.

"i no me correr á mi menos obligazion por Español i Castellano, sino antes mas que á otros, que profeso el estudio, i enseñanza de las lenguas, en que se echa mas de ver lo que esto inporta, para hazer este bien á mi nazion, i lengua natural, i dar claridad en escrivir i leer á nuestra xuventud i suzesores" (1954: 12).

\section{Un nuevo alfabeto para aprender a leer}

Sin duda, la ortografía de Correas se recuerda por su radical propuesta de reforma del alfabeto español bajo un estricto criterio fonético: una letra para cada sonido; para cada sonido que percibía el catedrático de Salamanca en unos años en los que los diferentes grados del reajuste consonántico del castellano hacían inviable un acuerdo de reforma fonética de la ortografía. El análisis del alfabeto de veinticinco letras demuestra que, aunque hubiese sido posible un acuerdo ortológico imprescindible para la ortografía fonética, el alfabeto de Correas no era perfecto (Bustos Tovar, 1998) ya que mantiene la oposición entre $b$ y $v$, la $h$ - inicial en recuerdo de la aspiración y no reconoce la consonante que se representa con $y$.

Ya desde el Arte de la lengua española castellana considera una de las mayores imperfecciones del español que no se pueda realizar una lectura fonética, independiente del significado de las palabras y los textos.

"solamente á servido la sinificazion i conozimiento de los vocablos para hechar de ver adonde servia de vocal, i que es consonante quando hiere á otra vocal con el sonido de va que la doi por nonbre, i ansi es menester saber la lengua primero para saber leer, que es suma inperfezion i cosa dina de remedio" (1954: 67).

Este defecto del idioma se soluciona con la reforma profunda del alfabeto desde un criterio fonético. En un alarde de optimismo injustificado, considera que su ortografía acortará el tiempo que los niños emplean en aprender a leer a un máximo de dos meses.

"De donde por no rretener nosotros el propio Abezedario Español se siguió que una letra hiziese dos oficios, i dos, i aun tres, uno, i se inuentasen çerilla, i tilde, i otros rremedios desacomodados que ai, sin saberse quien aia sido el inuentor, para suplir la falta: que es grandisimo enbarazo i dificultad para los que deprenden á leer. Porque siendo cosa de quinze ó veinte dias en xuizios algo capazes, ó de un mes, i a lo sumo de dos en los niños mas tiernos, si uviese buena ortografia" (1954: 10).

Más adelante el plazo se acorta aún más "será mui fazil enseñar i deprender á leer en pokos días" (1954: 120). El argumento pedagógico pasa a un primer plano en la disputa ortográfica y se convierte en un motivo recurrente en las ortografías españolas; la defensa de la ortografía fonética, además de la autoridad de Quintiliano y de Nebrija, se centra en que un alfabeto fonético permite aprender a leer con más celeridad y más facilidad que el alfabeto tradicional etimológico.

Es muy significativo cómo, a partir de 1630, los detractores de Alemán y de Correas emplean el mismo argumento pedagógico, pero en el sentido inverso; las novedades dificultan y retrasan el aprendizaje de la lectura precisamente porque rompen con la tradición. El culto sevillano (1631) de Juan de Robles inicia la reacción contra las propuestas de Correas tan solo un año después de la publicación de su ortografía donde propone el nuevo abecedario, y se censuran 
las nuevas letras en español con el mismo argumento que emplean quienes las defienden. Las ideas pedagógicas (no podía ser de otra manera) se habían incorporado definitivamente al debate ortográfico.

"Pues lo peor de todo es que no es esto lo más malo, que otra introdución se intenta peor sin conparación que la pasada de quitar i poner letras al $\mathrm{ABC}$, con otras mudanças de su forma i nonbre, [...] será fuerça que los que oi saben leer aprendan de nuevo con nuevo trabajo, i los que aprendieron con los nuevos, estrañen los antiguos i hayan de aprender segunda vez a leerlos con doblado trabajo i dilación, de forma que los niños salgan de las escuelas barbados, i hallen a sus padres en los hospitales, i los Maestros queden ricos, porque ningún médico se perdió jamás por ser larga la cura del enfermo" (1631: 227).

La diferencia entre los pocos días de Correas y las décadas de Robles demuestra que ambos exageran en la tradición de los polemistas de nuestro siglo XVII, Correas es recordado por su "terquedad, y aun su soberbia" (Bustos Tovar, 1998: 41) mientras que Robles se apunta en defensa vehemente de la tradición a cuantas polémicas se generan en estos años, como indica Gómez Camacho en su edición (1992); pero este apasionamiento es un ejemplo claro sobre cómo el argumento pedagógico pasa a ser fundamental en las ortografías del Seiscientos a partir de Mateo Alemán.

Es evidente que el problema con el alfabeto estaba muy relacionado con los problemas en la enseñanza de la lectura. Viñao Frago (1992) describe con precisión cómo era el método habitual para aprender a leer en el siglo XVII, que se iniciaba con el deletreo del alfabeto en mayúscula y en minúscula conociendo cada letra por su nombre, para continuar con el silabeo siempre en voz alta, un método común en el ámbito europeo (Chartier, 2004). De ahí la importancia capital de los abecedarios y los silabarios en las cartillas de enseñanza (Infantes, 2003), y la insistencia de Correas en que la autoridad real impusiese sus reformas a maestros e impresores; en el mismo contexto debemos entender su empeño en cambiar el nombre de las letras. Solo después de haber adquirido la competencia en la lectura de todas y cada una de las letras y las sílabas del español, se pasaba a la lectura de corrido de palabras y textos.

En realidad, el retraso en la lectura no se debía tanto al alfabeto que se aprendía, sino que se originaba por una práctica didáctica mecánica muy poco eficiente. Ya a finales del siglo XVIII, José de Anduaga en su Arte de escribir por reglas y sin muestras describe la práctica de la lectura en la que los niños "están casi las tres horas deletreando o leyendo en voz alta" (1795: 66), según un método que dilata el aprendizaje.

"De este mal método nace que los muchachos se están uno, dos y tres años para solo aprender á leer, y que los mas, aun quando salen de la escuela al cabo de los tres, quatro y un seis años, leen titubeando, con tonillo y generalmente sin dar sentido á lo mismo que leen" (1795: 177).

Dado que el método de enseñanza de la lectura partía de la memorización ordenada del abecedario y de las sílabas que se formaban (Infantes, 2003), Correas insiste en la necesidad de un nuevo orden más lógico que facilite el aprendizaje. Desde el punto de vista de la fonología, la propuesta es impecable y se basa en las posibilidades articulatorias para formar sílabas (Bustos Tovar, 1998).

\section{Conclusiones}

La polémica ortográfica de los Siglos de Oro provocada por los defensores de una reforma fonética de nuestra escritura, entre los que destacan Mateo Alemán y Gonzalo Correas, tuvo como consecuencia la incorporación de argumentos y reflexiones pedagógicas sobre la 
enseñanza de la lectura y la escritura que hasta hoy no han merecido la atención de la bibliografía especializada, más atenta a los aspectos filológicos de estas obras. La influencia de Mateo Alemán en la ortografía de Correas es muy evidente, aunque no recoge las ideas pedagógicas más importantes del autor del Guzmán de Alfarache que se podrían resumir en el aprendizaje conjunto de la lectura y escritura, la simplificación de la caligrafía para aprender a escribir y la crítica a la práctica didáctica de los maestros.

Gonzalo Correas incorpora de forma definitiva lo que hemos llamado el argumento pedagógico en la defensa de la ortografía fonética; como consecuencia las ortografías españolas a partir de 1630 hacen referencias a la educación, y a la enseñanza de la lectura y la escritura. Los detractores de las propuestas de Correas perciben con claridad la importancia de las referencias a la enseñanza en la argumentación que defiende la ortografía fonética e incorporan el criterio pedagógico como contraargumento para atacarla, en defensa de la ortografía tradicional.

El criterio pedagógico que busca facilitar el aprendizaje de los niños lleva a Correas a elaborar un nuevo alfabeto de veinte consonantes simples, en el que se suprimen e inventan letras renunciando a la tradición latina. También se proponen nuevos nombres para las letras y un silabario que combina los fonemas del español según sus posibilidades articulatorias y la frecuencia de aparición en la lengua. El objetivo principal de estas novedades es el aprendizaje de la lectura en un tiempo muy breve y con un método natural.

A partir de Correas, la polémica ortográfica del siglo XVII pasa a ser, además de una disputa filológica, fonética y lingüística, una reflexión pedagógica sobre cómo se enseñaba a leer y a escribir a los niños de nuestro Siglo de Oro.

\section{Referencias bibliográficas}

Alarcos García, E. (1919). Datos para la biografía de Gonzalo Correas. Boletín de la Real Academia Española, XXIX, 524-551.

Alemán, M. [1609] (1950). Ortografía castellana, edición de José Rojas Garcidueñas. México: Colegio de México.

Alemán, M. [1599/1604] (2012). Guzmán de Alfarache, edición de Luis Gómez Canseco. Madrid: Real Academia Española.

Anduaga, J. (1795). Arte de escribir por reglas y sin muestras. Madrid: Imprenta Real.

Baranda, N. (1995). Escritos para la educación de los nobles, siglos XVI y XVII. Bulletin Hispanique, 97, 157-171.

Bustos Tovar, J. (1998). Las propuestas ortográficas de Gonzalo Correas. Dicenda. Cuadernos de Filología Hispánica, 16, 41-62.

Cavillac, M. (1980). Mateo Alemán et la modernité: l'Ortografía castellana (1609). Bulletin Hispanique, 82, 380-401.

Chartier, A. M. (2001). La enseñanza de la lectura: un enfoque histórico, en Martínez Moctezuma, L. (coord.) La infancia y la cultura escrita. México: Siglo XXI, 147-190.

Chartier, A. M. (2004). Enseñar a leer y escribir: una aproximación histórica. México: Fondo de Cultura Económica.

Correas, G. [1625] (1954). Arte de la lengua española castellana, edición de Emilio Alarcos García. Madrid: CSIC.

Correas, G. (1627). Trilingue de tres artes de las tres lenguas Castellana, Latina, i Griega. Salamanca: Antonia Ramírez. 
Correas, G. [1627] (1984). Arte kastellana, edición de Manuel Taboada Cid. Santiago de Compostela: Universidad de Santiago de Compostela.

Correas, G. (1630). Ortografia kastellana nueva y perfeta. Salamanca: Xacinto Tabernier.

Esteba Ramos, D. (2005). La enseñanza del español en el Siglo de Oro: algunas consideraciones en torno al léxico. Interlingüística, 16 (1), 371-379.

Esteve Serrano, A. (2007). Contribución al estudio de las ideas ortográficas en España. Tonos. Revista electrónica de estudios filológicos, 13 [en línea]. Disponible en: http://www.um.es/tonosdigital/ znum13/secciones/relecturas_A_ideas.htm [consulta: 2013, 28 de mayo]

Frago, J. A. (2012). Razones de las reformas ortográficas en la América independiente y causas de su fracaso. Boletín de Filología, XLVII (2), 11-46.

Galán Melo, S. (1992). Ideas ortográficas en Gonzalo Correas, en M. Ariza, R. Cano, J. M. Mendoza y A. Narbona (eds.), Actas del II Congreso Internacional de Historia de la Lengua Española, I (pp. 265-272). Madrid: Pabellón de España.

Gómez Camacho, A. (2014). Las ideas pedagógicas en la Ortografía castellana de Mateo Alemán, Revista española de pedagogía, 257, 159-173, en prensa.

Hernando Cuadrado, L. (2010). El tratamiento de las partes de la oración en el Arte de la lengua española castellana de Gonzalo Correas. Boletín de Filología, XLV (2), 259-278.

Infantes, V., y Martínez, A. (2003). De las primeras letras. Cartillas españolas para enseñar a leer del siglo XVII. Salamanca: Ediciones Universidad de Salamanca.

Johnston, M. D. (1988). Mateo Alemán's Problem with Spelling. Publications of the Modern Language Association of America, CIII, 759-769.

Laspéras, J. M. (1995). Manuales de educación en el Siglo de Oro. Bulletin Hispanique, 97 (1), 173185.

Lope Blanch, J. M. (1997). La enseñanza del español durante el Siglo de Oro, en M. Fernández, F. García, y N. Vázquez (eds.), Actas del I Congreso Internacional de Historiografía Lingüística Española (pp. 49-74). Madrid: Arco Libros,

Martínez Marín, J. (1992). La evolución de la ortografía española: de la ortografía "de las letras" a la ortografía "de los signos de la escriturs", en M. Ariza, R. Cano, J. M. Mendoza y A. Narbona (eds.), Actas del II Congreso Internacional de Historia de la Lengua Española, I (pp. 753-761). Madrid: Pabellón de España.

Ortega Sánchez, D. (2011). Infancia, familia y educación en la Edad Moderna española: un recorrido a través de las fuentes pedagógicas (siglos XVI-XVIII). Tejuelo, 11, 85-103.

Parrack, J. C. (2005). The Picaresque School of Learning: Modernity and the Critique of Classical Humanism in Guzmán de Alfarache and the Ortografía castellana. Romance Notes, 45, 293-302.

Paz, Y. (2002). La Ortografía castellana de Mateo Alemán. Neophilologus, 86, 57-64.

Quetgles Roca, M. L. (2004). La educación en el Quijote. Revista de educación, nº . ext. 1, 119-137.

Robles, Juan de [1631] (1992). El culto sevillano, edición de Alejandro Gómez Camacho. Sevilla: Universidad de Sevilla.

Rodríguez San Pedro, L. E. (1986). El humanista Gonzalo Correas y su biblioteca salmantina (1631). Apunte valorativo. Studia historica, 4, 93-102.

Ruiz Berrio, J. (2004). El oficio de maestro en tiempos de Cervantes. Revista de educación, núm. ext. 1, 11-26.

Viñao Frago, A. (1992). Alfabetización, lectura y escritura en el Antiguo Régimen (siglos XVI-XVII), en A. Escolano (ed.), Leer y escribir en España. Doscientos años de alfabetización (pp. 45-68). Madrid: Fundación Germán Sánchez Ruipérez.

Viñao Frago, A. (1999). Alfabetización y primeras letras (siglos XVI-XVII) en Castillo, A. (comp.) Escribir y leer en el siglo de Cervantes. Madrid: Gedisa, 39-84. 
Viñao Frago, A. (2002). La enseñanza de la lectura y la escritura: Análisis socio-histórico. Anales de Documentación, 5, 345-359.

\begin{abstract}
I write as i talk: pedagogical ideas of Gonzalo Correas

INTRODUCTION. Gonzalo Correas is considered one of the main humanists in the Spanish Golden Century. The most prominent of his works is the Ortografia kastellana nueva i perfeta (1630), full of educational passages. METHODS. This paper focuses on the pedagogical ideas in Correas's works and the relationships between phonetic spelling and teaching of reading by means of comparative analysis between Correas's works and other Spanish orthographic works which used pedagogical arguments. They were interested in spelling that facilitate children learning although irreconcilable proposals were maintained. RESULTS. By examining Correas's pedagogical references, one prominent idea is emphasized: Spanish language demands a new alphabet that can be adapted to the seventeenth century pronunciation. The new letters would help Spanish children to learn and read faster and better than the traditional alphabet. Letter names and alphabetical order were also changed by Correas to ease learning. Accordingly, a new syllabary is proposed too. DISCUSSION. The results of this paper show that pedagogic arguments are incorporated into the controversy over Spanish orthography reform around the first third of the seventeenth century. These works, as well as linguisticand philological value, offer a valuable testimony about how literacy is conceived in the Spanish Golden Century that until now has not been studied.
\end{abstract}

Keywords: Gonzalo Correas, Spanish spelling, history of education, teaching reading, XVII century.

\title{
Résumé
}

J'écris comme je parle. Les idées pédagogiques de Gonzalo Correas

INTRODUCTION. Gonzalo Correas est considéré comme un des humanistes les plus importants du Siècle d'Or Espagnol; L'ouvre Ortografia kastellana nueva i perfeta est la plus connue et elle inclut une grande quantité de références éducatives. Ce travail analyse autant les idées pédagogiques de l'œuvre de Correas, que les relations qui s'établissent entre l'orthographie phonétique et l'enseignement de la lecture. MÉTHODE. Pour cela, on fait référence à l'analyse comparée des œuvres de Correas et des références à l'éducation des autres orthographies espagnoles du 17ème siècle, qui ont utilisé des arguments pédagogiques, toutes intéressées par une norme orthographique qui faciliterait l'apprentissage des enfants même si elles gardaient des propositions irréconciliables dans l'écriture. RÉSULTATS. En étudiant les références de l'éducation, nous voyons qu'une même idée se répète: l'espagnol a besoin d'un nouvel alphabet qui reflète la réalité de la prononciation au $17^{\text {ème }}$ siècle. Les nouvelles lettres aideront les enfants à apprendre la lecture d'une façon plus adéquate et plus rapide; Correas change non seulement le nom des lettres mais également l'ordre de l'alphabet pour faciliter l'apprentissage: Par conséquent, on proposerait des nouvelles syllabes. DISCUSSION. Le résultat de ce travail nous montre que l'argument pédagogique, 
pendant le premier tiers du $17^{\text {ème }}$ siècle, s'est incorporé définitivement à la controverse sur la réforme de l'orthographie espagnole. Ces ouvres, en plus de leur valeur linguistique et philologique, offraient un témoignage précieux sur la considération de l'alphabétisation pendant le Siècle d'Or espagnol qui, jusqu'à présent n'a pas été étudié.

Mots clés: Gonzalo Correas, Orthographie espagnole, Histoire de l'éducation, Enseignement de la lecture, $17^{\text {eme }}$ siècle.

\section{Perfil profesional del autor}

\section{Alejandro Gómez Camacho}

Profesor del Departamento de Didáctica de la Lengua y la Literatura y Filologías Integradas de la Facultad de Ciencias de la Educación de la Universidad de Sevilla. Ha centrado sus líneas de investigación en la norma lingüística en la comunicación electrónica, la adquisición de la competencia ortográfica en la educación secundaria y superior, el proyecto lingüístico de centro y sus repercusiones en el área de lengua española y literatura, y en las ideas pedagógicas en las ortografías y preceptivas del Siglo de Oro. En la actualidad, es el investigador responsable del grupo de investigación HUM 529 "Lengua española aplicada a la enseñanza", de la Universidad de Sevilla.

Correo electrónico de contacto: agomez21@us.es

Dirección para la correspondencia: Departamento de Didáctica de la Lengua y la Literatura y Filologías Integradas, Facultad de Ciencias de la Educación, c/ Pirotecnia, s.n. 41013 Sevilla. 